\title{
EBER (Irène), WAN (Sze-kar), WALF (Knut), eds., en collaboration avec MALEK (Roman), Bible in Modem China. The Literary and Intellectual Impact
}

Sankt Augustin (AU.), Institut Monumenta Serica, Jérusalem, The Harry S. Truman Research Institute for Advancement of Peace, The Hebrew University, 1999, 450 p. (illustr., index) (coll. « Monumenta Serica Monograph Séries » XLIII)

\section{Françoise Aubin}

\section{OpenEdition}

\section{Édition électronique}

URL : http://journals.openedition.org/assr/20633

DOI : $10.4000 /$ assr.20633

ISSN : $1777-5825$

\section{Éditeur}

Éditions de l'EHESS

\section{Édition imprimée}

Date de publication : 1 juillet 2000

Pagination : 114

ISBN : 2-222-96691-4

ISSN : 0335-5985

Référence électronique

Françoise Aubin, «EBER (Irène), WAN (Sze-kar), WALF (Knut), eds., en collaboration avec MALEK (Roman), Bible in Modem China. The Literary and Intellectual Impact », Archives de sciences sociales des religions [En ligne], 110 | avril-juin 2000, document 110-59, mis en ligne le 19 août 2009, consulté le 21 septembre 2020. URL : http://journals.openedition.org/assr/20633 ; DOI : https://doi.org/10.4000/ assr.20633

Ce document a été généré automatiquement le 21 septembre 2020.

(C) Archives de sciences sociales des religions 


\section{EBER (Irène), WAN (Sze-kar), WALF (Knut), eds., en collaboration avec MALEK (Roman), Bible in Modem China. The Literary and Intellectual Impact}

Sankt Augustin (AU.), Institut Monumenta Serica, Jérusalem, The Harry S. Truman Research Institute for Advancement of Peace, The Hebrew University, 1999, 450 p. (illustr., index) (coll. « Monumenta Serica Monograph Séries » XLIII)

\section{Françoise Aubin}

\section{RÉFÉRENCE}

EBER (Irène), WAN (Sze-kar), WALF (Knut), eds., en collaboration avec MALEK (Roman), Bible in Modem China. The Literary and Intellectual Impact, Sankt Augustin (AU.), Institut Monumenta Serica, Jérusalem, The Harry S. Truman Research Institute for Advancement of Peace, The Hebrew University, 1999, 450 p. (illustr., index) (coll. « Monumenta Serica Monograph Séries » XLIII)

1 Les traductions de la Bible dans des langues autochtones sont d'ordinaire envisagées comme un rouage de l'apostolat protestant en pays de mission. La présente publication de Monumenta Serica (une institution d'études sinologiques animée par les pères steylistes ou SVD, près de Bonn, et dirigée par le P. R. Malek, un éditeur exigeant et un missiologue entreprenant) est d'une tout autre envergure. Le colloque qui lui a donné naissance s'est tenu à Jérusalem en 1996, et il a été patronné par un bel assortiment oecuménico-savant d'instituts d'études hébraïques, bibliques, théologiques et d'instituts catholiques à vocation missionnaire, tandis que les collaborateurs venaient 
des horizons les plus variés (on aurait aimé mieux connaître leurs affiliations universitaires ou académiques). Le thème en était la Bible comme composante des rapports entre la Chine et l'Occident, et considérée le plus possible du point de vue chinois, selon la nouvelle tendance de la missiologie sinologique qui privilégie les versions chinoises du message chrétien et les réactions qu'il a suscitées (cf. Arch. supra $\left.\mathrm{n}^{\circ} 15\right)$.

2 Trois étapes de la transmission de la Bible en Chine ont été jaugées, à commencer par la traduction. Les obstacles catholiques à la divulgation populaire des Ecritures Saintes, alors que l'accent était mis sur la catéchèse et la pratique sacramentelle, ont fait que la première traduction catholique complète n'a pu aboutir avant 1968 (selon les belles contributions du jésuite Nicolas Standaert sur la transmission de la Bible dans la Chine du XVII siècle, pp. 32-54, et du franciscain Arnulf Camps sur l'entreprise du P.G.M. Allegra, ofm, le fondateur de Studium Biblicum Franciscanum, et son œuvre de traducteur, pp. 55-76). Chez les protestants, la préparation d'une traduction in extenso a été, au contraire, un souci permanent dès 1807 , avant même que les missionnaires puissent pénétrer dans le pays ; mais elle a été une source de conflits amers, d'une part entre les dénominations présentes en Chine, d'autre part entre les défenseurs d'un niveau ou d'un autre de la langue de traduction - langue parlée, langue écrite simple, haute langue littéraire. De telle sorte qu'elle s'est développée en plusieurs éditions rivales, jusqu'à une Union Version, dont la publication n'a pu être achevée qu'en 1934 (une communication nuancée et bien informée de Jost Zetzsche, pp.77-99). Les difficultés intrinsèques au travail de traduction proviennent d'abord des oppositions structurelles - syntaxiques, lexicographiques, rhétoriques, symboliques - entre l'hébreu et le chinois, ce que le linguiste Lihi Yariv-Laor (pp. 101-121) illustre par des exemples pratiques. À l'inverse, Knut Walf (pp.123-133) souligne les distorsions involontaires dans les traductions en langues européennes des termes fondamentaux du taoïsme qu'ont introduites les missionnaires, seuls intermédiaires de la connaissance en ce domaine, avant que le taoïsme ne devienne en Occident une discipline scientifique autonome. La question qui a le plus divisé les protestants, celle du «terme ", c'est-à-dire du terme chinois propre à rendre le nom de Dieu sans trace de polythéisme, est passée minutieusement en revue par l'éditeur principal du recueil, I.E. (pp. 135-161, historique qu'il faudrait compléter par l'aspect catholique du dilemme, tel qu'exposé par le P.J. Dehergne, sj, dans Actes du IIIe Colloque international, Chantilly, 1980, Paris, Les Belles-Lettres, 1983, pp. 13-44).

3 La seconde étape de la transmission des Écritures Saintes a été celle de sa réception, qui est envisagée ici dans l'œuvre du plus ancien théologien protestant chinois, le pasteur cantonnais Ho Tsun-sheen, ou Ho Chin-shan en chinois standard (par Laureen Pfister, pp. 165-197). Des traductions protestantes de la Bible réalisées par deux groupes différents de Miao - des autochtones du sud de la Chine - révèlent que le système phonétique en usage dans la Chine républicaine des années vingt n'a pas pris, alors que le système d'écriture inventé au début du siècle par le missionnaire Samuel Pollard est maintenant, grâce au succès de la Bible en cette écriture, considéré comme indigène (Joakim Enwall, pp.199-234, avec illustr.). Enfin, I.E. signale, au cours de son introduction, des transpositions poétiques dans les premières versions des Dix Commandements (p. 19).

$4 \quad$ L'ultime étape de la transmission est celle de l'appropriation des symboles, des images et des thèmes de la Bible - du Nouveau Testament plutôt que de l'Ancien, et de la Croix 
- dans la littérature du pays d'accueil (L.S. Robinson. pp. 237-277 ; R.D. Findeisen, pp. 279-299 ; Marián Galik, pp. 301-320); dans des traductions faites par les Chinois euxmêmes, par exemple les Psaumes (Francis K.H. So, pp. 321-349), ou dans des débats théologiques entre théologiens autochtones, ainsi entre Wu Lei-ch'uan et T.C. Chao (Sze-kar Wan, pp. 351-382), ou dans les études bibliques de la Chine communiste postmaoïste (Gong Liang, pp.383-407). La contribution finale de Wolfgang Kublin (pp. 409-427) pose le problème de la notion d'imperfection en Chine et en Occident.

Chaque article est suivi de son résumé en chinois, indice qu'un public savant est visé outre-mer ; et l'index, très complet comme tous ceux que compose le P. R. Malek, offre tous les caractères chinois qui parfois font défaut dans le corps du texte. 